Alma Mater Studiorum - Università di Bologna DEPARTMENT OF ECONOMICS

\title{
On Globally Optimal Punishments in the Repeated Cournot Game
}

Flavio Delbono

Luca Lambertini

Quaderni - Working Paper DSE N¹091

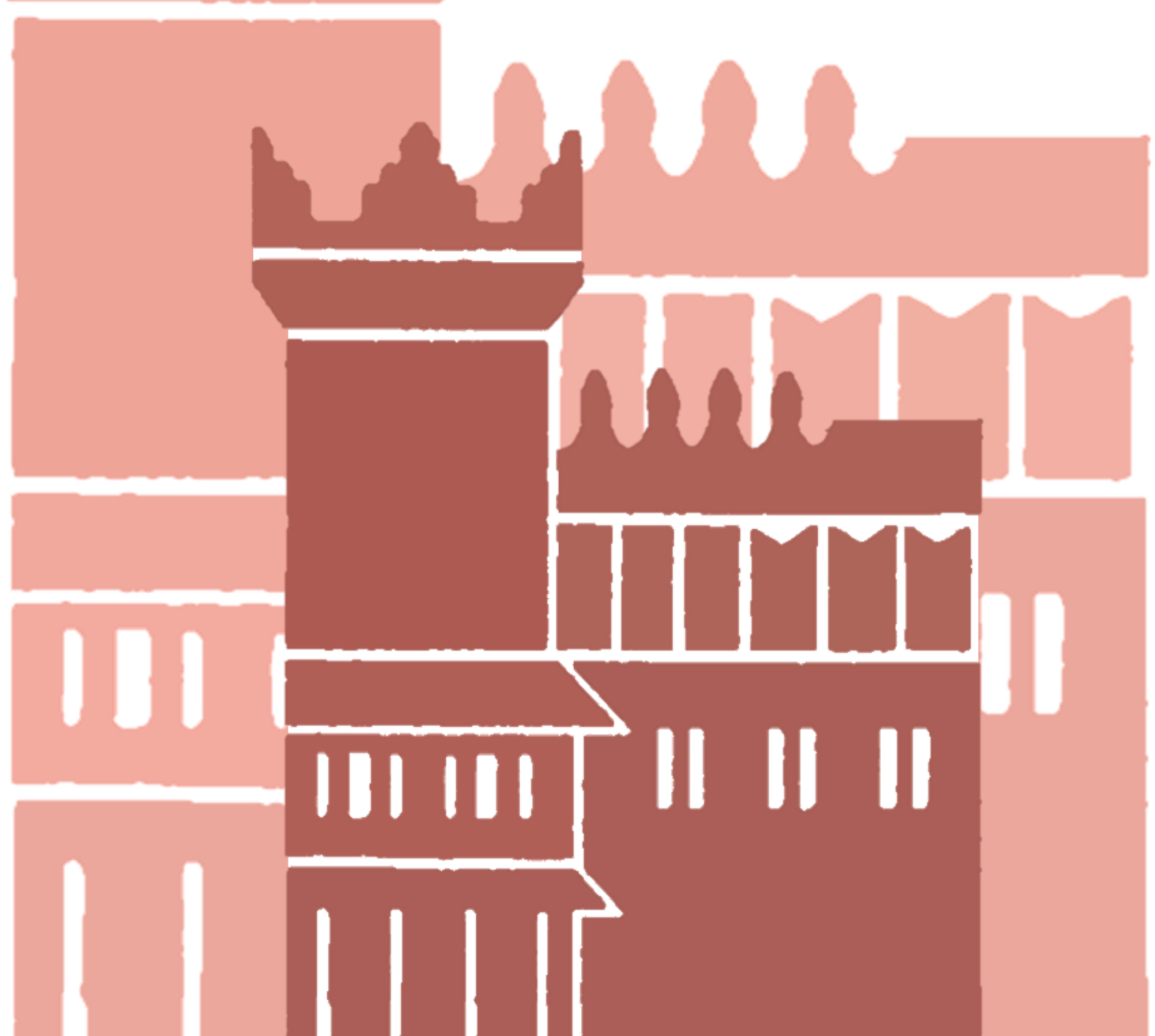




\title{
On Globally Optimal Punishments in the Repeated Cournot Game
}

\author{
Flavio Delbono $\#$ and Luca Lambertini ${ }^{\S}$ \\ Department of Economics, University of Bologna \\ \# Piazza Scaravilli 2, 40126 Bologna, Italy; flavio.delbono@unibo.it \\ $\S$ Strada Maggiore 45, 40125 Bologna, Italy; luca.lambertini@unibo.it
}

December 20, 2016

\begin{abstract}
We challenge the global optimality of one-shot punishments in infinitely repeated games with discounting. Specifically, we show that the stick-and-carrot punishment à la Abreu (1986) may not be globally optimal. We prove our result by investigating tacit collusion in the infinite repetition of a linear Cournot game. We illustrate the existence of the stick-and-carrot globally optimal punishment for large cartels, and fully characterise it. Then, we show that for mall cartels, global optimality may be reached only with two-period punishments.
\end{abstract}

Keywords: cartel stability, implicit collusion, repeated games

JEL Classification: C73, L13 


\section{Introduction}

While it is still an empirical question of major importance to establish if (and how) enterprises do collude, the theory of supergames has certainly strengthened our understanding of how cartels may be shaped by oligopolistic interaction. The term supergame was likely coined by Luce and Raiffa (1957) when examining repetitions of the prisoner's dilemma. Actually, according to Aumann (1981), "it has been known since the middle to late 1960s that any individually rational payoff vector (i.e., not smaller than a payoff that the player can guarantee himself) can be supported as a Nash equilibrium outcome in an infinitely repeated game where there is no discounting ... , and it is this result that he dubs the Folk Theorem" (Friedman 1986, p. 103).

However, when switching to impatient players, the strategies forming a subgame perfect equilibrium (SPE) in supergames without discounting do not necessarily work. In a pioneering paper, Friedman (1971) proved that every feasible payoff that Pareto dominates a Nash equilibrium of the stage game is a SPE payoff of the infinite repetition of the stage game, if players are patient enough. Friedman's punishment take the simple form of reversion to the Nash equilibrium of the stage game forever (grim strategy).

This line of research has been furthered by Abreu (1986). He identifies a class of (pure) strategies that support the Folk Theorem in repeated games with discounting. Such strategies allow one to find SPE where any individually rational outcome can be achieved. Moreover, Abreu (1986) has characterized a two-phase punishment as an optimal symmetric punishment which is more severe than Nash reversion; such a two-phase punishment consists of a stick-and-carrot strategy. Fudenberg and Maskin (1986) have then extended Abreu's results also to games of incomplete information. In Shapiro's (1989) words: "Within the class of symmetric punishments, Abreu proves that the optimal punishment has a simple, two-phase strategy: immediately following 
the defection, each firm participates in a 'price war' by producing a higher output than previously; but immediately thereafter all firms return to their optimal, tacitly-collusive output levels. It is striking that, when optimally punishing a defector, the industry returns after only a single period to the most collusive sustainable configuration. Abreu describes these types of punishments as offering a stick and a carrot; apparently, the carrot (returning to collusion) is necessary to make the stick (the one-period price war) both credible and as menacing as possible" (Shapiro, 1989, p. 368, italics added). Abreu (1986) also provides conditions under which the symmetric two-phase punishment is globally optimal.

In this paper we challenge such conditions showing that stick-and-carrot punishments may not be globally optimal and more than a single period is then required to enforce a collusive path. As a workhorse we employ the textbook version of a linear Cournot model as in Abreu's (1986, p. 206) example. We know that: (i) global optimality of punishments requires that "in continuation equilibria firms earn zero profits" (Shapiro, 1989, p. 369); (ii) condition (i) is granted by minmax strategies after deviation from the cartel, and (iii) minmax strategies are nor subgame perfect in variable-sum games as ours. Hence, the central issue we are going to tackle deals with the existence of subgame perfect punishments capable of reproducing the same critical threshold of the discount factor as under minmax strategies. We prove that, for small cartels, stick-and-carrot is not a globally optimal strategy and global optimality can be reached only via two-period punishments. This result belies the validity of Abreu's (1986) Theorems 18 and 19. For large cartels, instead, a stick-and-carrot punishment is globally optimal as it entails the same critical threshold of the discount factor as under minmax. Irrespective of cartel size, his example is mistaken.

The paper is organized as follows. In Section 2 we present the setup and establish the benchmark for the threshold of the discounted factor under 
grim or minmax strategies. In Sections 3 and 4 we get into Abreu's analysis and investigate the optimality of stick-and-carrot punishments. In Section 5 we show under which conditions, for small cartels, global optimality may be obtained by means of a non stationary punishment lasting two periods. Section 6 concludes.

\section{Setup}

Consider a market for a homogeneous good, served by $\mathcal{N}=2, \ldots n$ identical single-product firms, endowed with the same technology. Let the market exist over discrete time $t=0,1,2, \ldots \infty$. All firms share the same intertemporal preferences, measured by the time-invariant discount factor $\delta \in[0,1]$. In each period, the inverse market demand function is

$$
p=a-\sum_{i=1}^{n} q_{i}
$$

where parameter $a>0$. The cost function of firm $i$ is $C_{i}=c q_{i}$ with $c \in[0, a)$. Accordingly, the individual profits are

$$
\pi_{i}=\left(a-\sum_{i=1}^{n} q_{i}-c\right) q_{i}
$$

\subsection{Grim trigger and minmax strategies}

Consider first Friedman's (1971) version of the grim trigger strategies, where collusion is sustained by the threat of an infinite reversion to the CournotNash equilibrium of the constituent game. We briefly summarise this result here. Assume perfect tacit collusion with cartel members setting the output vector so as to maximise joint profits $\Pi=\sum_{i=1}^{n} \pi_{i}$. The resulting individual collusive output level is $q^{C}=(a-c) /(2 n)$, granting an individual profit 
$\pi^{C}=(a-c)^{2} /(4 n)$. The unilateral deviation against the $n-1$ loyal cartel members is

$$
q^{D}\left(q^{C}\right)=\frac{(a-c)(n+1)}{4 n}
$$

delivering deviation profits

$$
\pi^{D}\left(q^{C}\right)=\frac{(a-c)^{2}(n+1)^{2}}{16 n^{2}}
$$

During the infinite Nash reversion, the per-period Cournot-Nash profits are $\pi^{N}=(a-c)^{2} /(n+1)^{2}$. Collusion is stable iff

$$
\delta \geq \frac{\pi^{D}-\pi^{C}}{\pi^{D}-\pi^{N}} \equiv \delta_{F}=\frac{(n+1)^{2}}{n(n+6)+1}
$$

where $\delta_{F}$ is concave and monotonically increasing in $n$, with $\delta_{F}=9 / 17$ for $n=2$ and $\lim _{n \rightarrow \infty} \delta_{F}=1$. Treating $n$ as a continuous variable,

$$
\frac{\partial \delta_{F}}{\partial n}=\frac{4\left(n^{2}-1\right)}{[n(n+6)+1]^{2}}>0
$$

The intuitive message, which has been incorporated in the acquired view on these matters, is that, in the collusive outcome "the per period and per firm profit is a decreasing function of $n$. A large number of firms reduces the profit per firm and thus the cost of being punished for undercutting. In contrast, the short run gain from undercutting the monopoly price slightly... increases with $n \ldots$ In this sense market concentration facilitates tacit collusion" (Tirole, 1988, p. 248).

If instead the infinite punishment consists in each player reverting to minmax one another through $q_{m}=(a-c) / n$, the per-period individual payoff during the punishment phase is nil and the threshold of the discount factor ensuring the stability of tacit collusion becomes

$$
\delta \geq \frac{\pi^{D}-\pi^{C}}{\pi^{D}} \equiv \delta_{m}=\frac{(n-1)^{2}}{(n+1)^{2}}<\delta_{F} \forall n
$$


where subscript $m$ mnemonics for minmax, with $\delta_{m}=1 / 9$ for $n=2$ and $\lim _{n \rightarrow \infty} \delta_{m}=1$. That is, minmax strategies appear to be more efficient than Nash ones in stabilising the cartel. However, since the constituent game is a variable-sum one, it is well known that the use of minmax strategies does not produce subgame perfection. Yet, the threshold of the discount factor $\delta_{m}$ delivered by minmax strategies identifies the benchmark to be reproduced using globally optimal punishments which must meet the additional requirement of subgame perfection.

\section{The supergame with optimal punishments}

Abreu (1986) aims at finding a one-shot punishment strategy possessing the properties of being (i) subgame perfect, (ii) more efficient than Friedman's (1971) Nash reversion, and (iii) globally optimal. Since the stick is harsher than the Nash strategy, this requires a condition of its own for incentive compatibility about the implementation of the punishment itself. Moreover, since the severity of the punishment may drive the resulting punishment profits below zero, even if for a single period, one has to control for the nonnegativity of the discounted flow of profits over the continuation of the game from the punishment period to doomsday. All of this requires the following conditions to hold in a symmetric subgame perfect equilibrium:

$$
\begin{gathered}
\pi^{D}\left(q^{C}\right)-\pi^{C} \leq \delta\left(\pi^{C}-\pi^{P}\right) \\
\pi^{D}\left(q^{P}\right)-\pi^{P} \leq \delta\left(\pi^{C}-\pi^{P}\right) \\
\pi^{P}+\pi^{C} \sum_{t=1}^{\infty} \delta^{t}=\pi^{P}+\frac{\delta \pi^{C}}{1-\delta} \geq 0
\end{gathered}
$$

considering, for the moment, $\pi^{D}\left(q^{P}\right)>0$. For the moment, we shall suppose it is. This amounts to saying that we start examining optimal (but not 
necessarily globally optimal) punishments, with conditions (8-10) referring to the setup in Abreu (1986) up to his Lemma 17, p. 204.

Inequality (8) must be satisfied for the collusive path to be stable. Inequality (9) must hold for firms to implement the optimal punishment $q^{P}$, delivering the punishment payoff $\pi^{P}, \pi^{D}\left(q^{P}\right)$ being the profits generated by the optimal deviation $q^{*}\left(q^{P}\right)$ from the punishment $q^{P}$. Condition (10) is the participation constraint whereby the discounted continuation payoff cannot be negative. Sticking to the assumption of full collusion, the two unknowns to be determined are the critical threshold of the discount factor and the intensity of the punishment $q^{P}$.

If all firms adopt the punishment, the per-firm punishment profits are

$$
\pi^{P}=\left(a-n q^{P}-c\right) q^{P}
$$

while the unilateral deviation from $q^{P}$ is

$$
q^{*}\left(q^{P}\right)=\frac{a-c-(n-1) q^{P}}{2}>0 \forall q^{P} \in\left(0, \frac{a-c}{n-1}\right)
$$

where, for future reference, we may define $(a-c) /(n-1) \equiv q^{P}$. Whenever the best reply in (12) is indeed positive, the profits granted by optimally deviating from the punishment are

$$
\pi^{D}\left(q^{P}\right)=\frac{\left[a-(n-1) q^{P}-c\right]^{2}}{4}
$$

Now, solving (8-9) w.r.t. $\delta$ and $q^{P}$, one obtains

$$
\begin{gathered}
\delta \geq \frac{(n+1)^{2}}{16 n} \equiv \delta_{A} \\
q^{P} \geq q_{A}^{P} \equiv \frac{(a-c)(3 n-1)}{2 n(n+1)}
\end{gathered}
$$

with $\partial q_{A}^{P} / \partial n<0$, for the intensity of the punishment is diluted as the number of cartel members increases. In correspondence of the lower bound of $q^{P}$ in 
(14), the optimal unilateral deviation from the punishment is

$$
q^{*}\left(q^{P}\right)=\max \left\{0, \frac{(a-c)[n(6-n)-1]}{4 n(n+1)}\right\}
$$

with

$$
\frac{(a-c)[n(6-n)-1]}{4 n(n+1)}>0 \forall n \in[2,5]
$$

In the same range of $n$,

$$
p\left(q^{*}\left(q^{P}\right), q^{P}\right)=a-\frac{(a-c)[n(6-n)-1]}{4 n(n+1)}-(n-1) q_{A}^{P}>0
$$

Leaving aside for a moment the fact that $n$ is an integer, observe that $n(6-n)-1=0$ in $n=3+2 \sqrt{2} \simeq 5.83$. This implies that the system of inequalities (8-10) is admissible only for $n \in[2,5]$. In this range, substituting $\delta_{A}, q^{C}$ and

$$
q^{*}\left(q^{P}\right)=\frac{(a-c)[n(6-n)-1]}{4 n(n+1)}
$$

into (10) reveals that the participation constraint does not bite.

Moreover, $\delta_{A}>\delta_{m}$ for all $n \in[2,5]$, revealing that

Lemma 1 For all $n \in[2,5]$, the stick-and-carrot punishment with $\pi^{D}\left(q^{P}\right)>$ 0 cannot be globally optimal.

Additionally, $\delta_{A}>\delta_{F}$ for all $n \geq 10$ and $\delta_{A}>1$ for all $n \geq 14$. Hence, we must abandon the idea that the optimal deviation from the punishment delivers positive profits and find a way towards the characterization of globally optimal punishments.

\section{Looking for globally optimal punishment}

The finding in Lemma 1 takes us to Abreu (1986), where the value of the discounted payoff flow generated by the continuation of the repeated game 
after the initial deviation from the collusive path appearing in (10) is defined as

$$
\pi^{P}+\frac{\delta \pi^{C}}{1-\delta} \equiv \mathbf{v}(\Gamma)
$$

Abreu (1986, p. 205) says that "... there exists a lower bound $\underline{\delta}<1$ $\ldots$ such that for $\delta \geq \underline{\delta}, \mathbf{v}(\Gamma)=0$. Since a firm's minmax payoff in the component game is zero, global optimality is clearly implied ... an interesting feature of the optimal two-phase punishment when $\mathbf{v}(\Gamma)=0$ is that all firms simultaneously minmax one another in the first phase". Then, his Theorems 18 and 19 rely upon the following argument: "The only way $\mathbf{v}(\Gamma)$ can equal zero and $\sigma\left(x_{1}, x_{2}\right)$ be a P.E. is $\pi^{*}\left(x_{1}\right)=0 \ldots$ Hence the total output produced by $(N-1)$ firms must be large enough that $p(N-1) x_{1} \leq c$, which sets a lower bound on $x_{1}$ independent of $\delta$. Hence profits in the first period must be negative, but not so large that they cannot be recouped by a collusive output level supportable by a zero punishment in the future."

In our model, $N=n, x_{1}=q^{P}, x_{2}=q^{C}$, and $\pi^{*}\left(x_{1}\right)=\pi^{D}\left(q^{P}\right)$. Moreover, $\sigma\left(x_{1}, x_{2}\right)=\sigma\left(q^{P}, q^{C}\right)$ is a symmetric strategy profile for the supergame based on the two-phase (stick-and-carrot) punishment. Finally, P.E. stands for perfect equilibrium.

Now, observe that the adoption of minmax strategies forever after the deviation from the cartel path ensures $\mathbf{v}(\Gamma)=0$ and therefore $\delta_{m}$ cannot be outperformed by any other form of punishment as this would require $\mathbf{v}(\Gamma)$ becoming negative, consequently driving firms out of the supergame. The issue is then whether there exists a subgame perfect punishment capable of reproducing $\delta_{m}$.

For the moment, we confine our attention to $n \in[2,5]$. Abreu (1986, p. 206) uses the same setting as ours to provide an example illustrating his Theorems 18-19. For $n \in[2,5]$, he claims that

$$
\underline{\delta}=\frac{4 n}{(n+1)^{2}} \equiv \delta_{25}
$$


A few remarks are in order:

- To begin with, compare $\delta_{25}$ against $\delta_{A}$ to find that

$$
\delta_{25}-\delta_{A}=\frac{[n(6-n)-1][n(n+10)+1]}{16 n(n+1)^{2}}>0
$$

meaning that, for small cartels, using $\mathbf{v}(\Gamma)>0$ is more efficient than imposing $\mathbf{v}(\Gamma)=0$.

- Secondly, and fairly surprising, in $n=2, \delta_{25}=8 / 9>\delta_{F}=9 / 17$. The same ranking between $\delta_{25}$ and $\delta_{F}$ emerges for $n=3,4$.

- Thirdly, $\delta_{25}$ is obviously decreasing in $n$. This would entail that enlarging the cartel makes it more stable, which goes against the acquired wisdom intuitively sustaining the opposite.

These observations prompt for a reconstruction of the example, which can be worked out as follows. Since the requirement is $\mathbf{v}(\Gamma)=0$, one can solve (10) at the margin w.r.t. $\delta$, to find

$$
\delta=\frac{4 n q^{P}\left(n q^{P}+c-a\right)}{\left(2 n q^{P}+c-a\right)^{2}}
$$

Then, solving (9) at the margin w.r.t. the punishment, we obtain $q^{P}=$ $(a-c) /(n-1)$. In correspondence of this intensity of the punishment, $q^{D}\left(q^{P}\right)=$ $0=\pi^{D}\left(q^{P}\right)$ (i.e., in Abreu's terminology, $\pi^{*}\left(x_{1}\right)=0$ ). Moreover, simplifying (22) yields $\delta_{25}$. At this point, using these expressions for $\delta$ and $q^{P}$, one may check that (8) is satisfied as an inequality for all $n \in[2,5]$.

This proves that solving the sub-system (8-9) and then checking (10) is not equivalent to solving sub-system (9-10) and then checking (8). The reason is that, if the deviation profits $\pi^{D}\left(q^{P}\right)$ and the continuation payoff are both nil, then necessarily (9) and (10) coincide, as can be easily verified rewriting (9) as

$$
-\pi^{P} \leq \delta\left(\pi^{C}-\pi^{P}\right) \Leftrightarrow \delta \pi^{C}+(1-\delta) \pi^{P} \geq 0
$$


which evidently coincides with (10) up to the positive constant $1 /(1-\delta)$.

We are dealing with three inequalities in two unknowns, $\delta$ and $q^{P}$. Hence, the reason why the two routes do not deliver the same conclusion is that, when $\pi^{D}\left(q^{P}\right)=0,(9)$ and (10) are indeed the same and therefore $\delta_{25}$ results from using a single condition to find both unknowns.

For $n \geq 6$, Abreu's example yields

$$
\underline{\delta}=\frac{(n+1)^{2}}{4(n-1)^{2}} \equiv \delta_{6}
$$

This also deserves a few comments:

- Comparing $\delta_{m}$ with $\delta_{6}$, we have

$$
\delta_{m}-\delta_{6}=\frac{[n(n-6)+1][n(3 n-2)+3]}{4(n+1)^{2}(n-1)^{2}}>0
$$

This would imply that the presumed optimal punishment outperforms minmax strategies, which is impossible.

- Moreover, $\delta_{6}$ monotonically decreases in $n$, with

$$
\lim _{n \rightarrow \infty} \delta_{6}=\frac{1}{4}<\left.\delta_{A}\right|_{n=2}=\frac{9}{32}
$$

which would suggest that an infinitely large cartel is more easily sustained than the smallest cartel, which again goes against intuition and acquired wisdom.

On the basis of Abreu's (1986) Theorems 18 and 19, globally optimality of the punishment requires $\mathbf{v}(\Gamma)=0$ and $\pi^{D}\left(q^{P}\right)=0$, which means that the system (8-10) becomes:

$$
\begin{gathered}
\pi^{D}\left(q^{C}\right)-\pi^{C} \leq \delta\left(\pi^{C}-\pi^{P}\right) \\
-\pi^{P} \leq \delta\left(\pi^{C}-\pi^{P}\right)
\end{gathered}
$$




$$
\pi^{P}+\pi^{C} \sum_{t=1}^{\infty} \delta^{t}=\pi^{P}+\frac{\delta \pi^{C}}{1-\delta} \geq 0
$$

As observed above, the last two inequalities are identical up to a positive constant. Hence, solving (27) and (28) or (27) and (29) necessarily yields the same pair:

$$
\begin{gathered}
\delta \geq \delta_{m} \equiv \frac{(n-1)^{2}}{(n+1)^{2}} \\
q^{P} \geq q_{m}^{P} \equiv \frac{(a-c) \sqrt{n}(\sqrt{n}+1)^{2}}{4 n^{3}}
\end{gathered}
$$

We have then shown that when (28) and (29) are equivalent, satisfying the individual incentive to implement the optimal punishment at the margin implies that the continuation value from that period onwards is exactly equal to zero and replicates the efficiency of the infinite reversion to the minmax strategy $q_{m}$ after the initial deviation from the cartel path. However, in order for (30) to be acceptable, it must be true that $\pi^{D}\left(q^{P}\right)=0$, which, in turn, requires $q^{*}\left(q^{P}\right)=0$, i.e., that the optimal deviation from the punishment be nil. Looking at the best reply

$$
q^{*}\left(q^{P}\right)=\frac{a-(n-1) q^{P}-c}{2}
$$

it appears that $a-(n-1) q^{P}-c \leq 0$ for all $q^{P} \geq \underline{q}^{P}$. Moreover, the resulting price $p\left(\underline{q}^{P}\right)=a-n \underline{q}^{P}$ can be lower than marginal cost but cannot drop below zero. The latter non-negativity condition requires $c \geq a / n$. The same constraint holds for $p\left(q_{m}^{P}\right)=a-n q_{m}^{P}$, requiring $c \geq a(\sqrt{n}-1)^{2} /(\sqrt{n}+1)^{2}$, with

$$
\frac{a(\sqrt{n}-1)^{2}}{(\sqrt{n}+1)^{2}}>\frac{a}{n} \forall n \geq 6
$$

Now, comparing $q_{m}^{P}$ with $\underline{q}^{P}$, we obtain

Lemma $2 q_{m}^{P}>\underline{q}^{P}$ for all $n \geq 6$. Therefore, $q^{*}\left(q^{P}\right)=\pi^{D}\left(q^{P}\right)=0$ for all $n \geq 6$. 
This Lemma entails two relevant consequences. The first is that $\delta_{6}<\delta_{m}$ is clearly a mistake. ${ }^{1}$ The second, more important implication of the above Lemma is

Proposition 3 For all $n \geq 6$, the one-shot optimal punishment is $q^{P} \geq q_{m}^{P}$, delivering the same threshold $\delta_{m}$ of the discount factor for the stability of full collusion as minmax strategies repeated forever.

We may then conclude that, for $n \in[2,5]$, the globally optimal one-shot punishment does not exists and, if $\delta_{m}$ can indeed be reached, it is the outcome of a punishment lasting at least two periods, and possibly non-stationary (as already illustrated in Abreu, 1988).

\section{Two-period punishment}

There are two alternative approaches to model a non-stationary punishment lasting two periods. One consists in stipulating that, once again, the optimal deviation from the punishment yields zero profits. If so, the relevant system is

$$
\begin{gathered}
\pi^{D}\left(q^{C}\right)-\pi^{C} \leq \sum_{t=1}^{2} \delta^{t}\left[\pi^{C}-\pi^{P}\left(q_{t}^{P}\right)\right] \\
-\pi^{P}\left(q_{1}^{P}\right) \leq \sum_{t=1}^{2} \delta^{t}\left[\pi^{C}-\pi^{P}\left(q_{t}^{P}\right)\right] \\
\pi^{P}\left(q_{1}^{P}\right)+\delta \pi^{P}\left(q_{2}^{P}\right)+\frac{\delta^{2} \pi^{C}}{1-\delta} \geq 0
\end{gathered}
$$

where $q_{t}^{P}$, with $t=1,2$ is the punishment in period $t$. Solving (33-35) w.r.t. $\left\{q_{1}^{P}, q_{2}^{P}, \delta\right\}$ yields

$$
q_{1}^{P}=q_{m}^{P} ; q_{2}^{P}=q^{C} ; \delta=\delta_{m}
$$

\footnotetext{
${ }^{1}$ We didn't succeed in reconstructing the derivation of $\delta_{6}$ in Abreu's (1986, p. 205) example. For sure, it cannot represent the minimal theshold of the discount factor, as it is lower than $\delta_{m}$.
} 
which proves that imposing $\pi^{D}\left(q_{1}^{P}\right)=0$ replicates the above (unacceptable) result whereby the individual output in the first period of the punishment is $q_{m}^{P}$ and then firms revert to the cartel production in the second period of the presumed punishment.

Hence, this approach cannot be pursued. The second approach consists in considering

$$
\pi^{D}\left(q_{1}^{P}\right)=\frac{\left[a-(n-1) q_{1}^{P}-c\right]^{2}}{4}>0
$$

together with a non-stationary punishment, as in Lambertini and Sasaki (2002). The related system of inequalities is

$$
\begin{gathered}
\pi^{D}\left(q^{C}\right)-\pi^{C} \leq \sum_{t=1}^{2} \delta^{t}\left[\pi^{C}-\pi^{P}\left(q_{t}^{P}\right)\right] \\
\pi^{D}\left(q_{1}^{P}\right)-\pi^{P}\left(q_{1}^{P}\right) \leq \sum_{t=1}^{2} \delta^{t}\left[\pi^{C}-\pi^{P}\left(q_{t}^{P}\right)\right] \\
\pi^{P}\left(q_{1}^{P}\right)+\delta \pi^{P}\left(q_{2}^{P}\right)+\frac{\delta^{2} \pi^{C}}{1-\delta} \geq 0
\end{gathered}
$$

Condition (38) reformulates the constraint concerning the stability of the collusive path, while (39) ensures players' incentive compatibility about the implementation of a biperiodal punishment. The third condition prevents players quitting the supergame. In all of them,

$$
\pi^{P}\left(q_{t}^{P}\right)=\left(a-2 q_{t}^{P}-c\right) q_{t}^{P}
$$

and, in (39-40),

$$
\pi^{D}\left(q_{t}^{P}\right)=\frac{\left[a-c-(n-1) q_{t}^{P}\right]^{2}}{4}, t=1,2
$$

The system (38-40) delivers four solutions w.r.t. the triple $\left\{q_{1}^{P}, q_{2}^{P}, \delta\right\}$, 
of which only one is acceptable:

$$
\begin{gathered}
q_{1}^{P} \geq q_{A}^{P} \equiv \frac{(a-c)(3 n-1)}{2 n(n+1)} \\
q_{2}^{P} \geq \widehat{q}_{2}^{P} \equiv \frac{(a-c)[2 n(n-1)+(1+n(n-6)) \sqrt{n}]}{4 n^{2}(n-1)} \\
\delta \geq \delta_{m} \equiv \frac{(n-1)^{2}}{(n+1)^{2}}
\end{gathered}
$$

On the basis of (43), one can easily establish

$$
\underline{q}^{P}>\frac{(a-c)(3 n-1)}{2 n(n+1)}>\frac{(a-c)[2 n(n-1)+(1+n(n-6)) \sqrt{n}]}{4 n^{2}(n-1)}
$$

for all $n \in[2,5]$, i.e., the punishment is decreasing over time and the constraint about the the optimal deviation from the punishment is respected.

Moreover, plugging the triple $\left\{q_{1}^{P}=q_{A}^{P}, q_{2}^{P}=\widehat{q}_{2}^{P}, \delta=\delta_{m}\right\}$ into (40), one finds that the constraint ensuring the firms' participation to the continuation of the supergame is indeed satisfied at the margin, i.e., (40) holds as an equality. Moreover, controlling for the price in the two punishment periods, it turns out that it is strictly positive for $n=2,3$, while for $n=4,5$, a sufficient condition for its non-negativity is $a \in(c, 7 c]$. To see this, observe that the price levels in the two punishment periods are

$$
\begin{gathered}
p\left(q_{1}^{P}\right)=\frac{c(3 n-1)-a(n-3)}{2(n+1)} \\
p\left(q_{2}^{P}\right)=\frac{c\left(1-2 \sqrt{n}-6 n+2 n^{3 / 2}+n^{2}\right)-a\left(1+2 \sqrt{n}-6 n-2 n^{3 / 2}+n^{2}\right)}{4 \sqrt{n}(n-1)}
\end{gathered}
$$

Whereas $p\left(q_{2}^{P}\right)>0$ for all $n \in[2,5], p\left(q_{1}^{P}\right)$ may become negative at $n=4,5$ because its partial derivative w.r.t. $a$ is negative for $n=4,5$.

The foregoing discussion can be summarised in the following:

Proposition 4 For $n \in[2,5]$, the globally optimal punishment requires two periods and its intensity is decreasing over time. It is admissible for all 
$a \in(c, 7 c]$. The resulting critical threshold of the discount factor for the stability of full collusion is the same as under infinite reversion to the minmax strategies, $\delta_{m}$, at which $\mathbf{v}(\Gamma)=0$.

On the basis of Propositions 3-4, we can formulate

Theorem 5 Assume $a \in(c, 7 c]$. In the linear Cournot supergame, there exists a globally optimal punishment path minimising the value of the stability threshold of the discount factor for all $n \geq 2$. For any cartel size, the discounted payoff flow generated by the continuation game following the initial deviation from the cartel path is nil. However, the structure of the globally optimal punishment depends on cartel size:

- the punishment is one-shot (stick-and-carrot) only for $n \geq 6$, in which case it requires the optimal deviation payoff from the punishment to be nil, without any restriction on market size;

- for smaller cartels, $a \in(c, 7 c]$ is required and the optimal deviation payoff from the punishment must not be nil. The punishment must be distributed over two periods along which its severity is decreasing.

Now recall Abreu's (1986, p. 205) claim "Consider two-phase punishments. The only way $\mathbf{v}(\Gamma)$ can equal zero and $\sigma\left(x_{1}, x_{2}\right)$ be a P.E. is if $\pi^{*}\left(x_{1}\right)=0 \ldots$.." as reported above. Our Theorem proves that the attainment of the lowest threshold of the discount factor $\delta_{m}$ does require $\mathbf{v}(\Gamma)=0$, but not, in general, $\pi^{*}\left(x_{1}\right)=0$. Indeed, for small cartels, this is not the case because the stick-and-carrot punishment scheme does not work, global optimality requiring a two-period punishment. The intuitive explanation relies upon the fact that the slice from cartel participation decreases with cartel size. Hence, the punishment required to stabilise a small cartel has to be more severe than for large cartels. This, when global optimality is looked for, entails an extension of the punishment span. 


\section{Concluding remarks}

In this paper, we have proved that global optimality in the infinite repetition of a linear Cournot game may not be granted by one-shot stick-and-carrot strategy. Our conclusions contrast strikingly with those of Abreu (1986). We show that globally optimal subgame perfect strategies depend on the number of firms. For 'large' cartels, the globally optimal one-shot punishment exists and reproduces the same critical threshold of the discount factor as under minmax strategies. For 'small' cartels, global optimality cannot be implemented via one-shot punishments, but only by means of a two-period punishment.

Whatever is the number of firms, the example in Abreu (1986) delivers thresholds of the discount factor which cannot be globally optimal as they do not coincide with that delivered by the reversion to the minmax strategy. The source of this problem is that the requirement that the payoff flow generated by the continuation of the supergame be nil makes two of the three incentive compatibility constraints coincide.

Our findings, which we have derived from the simplest possible framework, might prelude to a revisitation of the large literature investigating the impact of product differentiation on cartel stability ${ }^{2}$ using Friedman's (1971) folk theorem and the representative consumer with a preference for variety as in Singh and Vives (1984).

\footnotetext{
${ }^{2}$ See Deneckere (1983), Majerus (1988), Ross (1992), Rothschild (1992), Lambertini (1997) and Albæk and Lambertini (1998), inter alia.
} 


\section{References}

[1] Abreu, D.J. (1986), "Extremal Equilibria of Oligopolistic Supergames", Journal of Economic Theory, 39, 191-225.

[2] Abreu, D.J. (1988), "On the Theory of Infinitely Repeated Games with Discounting", Econometrica, 56, 383-96.

[3] Albæk, S. and L. Lambertini (1998), "Collusion in Differentiated Duopolies Revisited", Economics Letters, 59, 305-8.

[4] Aumann, R.J. (1981), "Survey of Repeated Games", in R. J. Aumann et al. (eds), Essays in Game Theory, Mannheim, Bibliographisches Institut.

[5] Deneckere, R. (1983), "Duopoly Supergames with Product Differentiation", Economics Letters, 11, 37-42.

[6] Friedman, J.W. (1971), "A Non-Cooperative Equilibrium for Supergames", Review of Economic Studies, 28, 1-12.

[7] Friedman, J.W. (1986), Game Theory with Applications to Economics, Oxford, Oxford University Press.

[8] Fudenberg, D. and e. Maskin (1986), "The Folk Theorem in Repeated Games with Discounting or with Incomplete Information", Econometrica, 54, 533-54.

[9] Lambertini, L. (1997), "Prisoners' Dilemma in Duopoly (Super)Games", Journal of Economic Theory, 77, 181-91.

[10] Lambertini, L. and D. Sasaki (2002), "Non-Negative Quantity Constraints and the Duration of Punishment", Japanese Economic Review, $53,77-93$. 
[11] Luce, R. D. and H. Raiffa (1957), Games and Decisions, New York, Wiley.

[12] Majerus, D. (1988), "Price vs Quantity Competition in Oligopoly Supergames", Economics Letters, 27, 293-7.

[13] Ross, T.W. (1992), "Cartel Stability and Product Differentiation", International Journal of Industrial Organization, 10, 1-13.

[14] Rothschild, R. (1992), "On the Sustainability of Collusion in Differentiated Duopolies", Economics Letters, 40, 33-7.

[15] Shapiro, C. (1989), "Theories of Oligopoly Behavior", in R. Schmalensee and R. Willig (eds), Handbook of Industrial Organization, vol. I, Amsterdam, North-Holland.

[16] Singh, N. and X. Vives (1984), "Price and Quantity Competition in a Differentiated Duopoly", RAND Journal of Economics, 15, 546-54.

[17] Tirole, J. (1988), The Theory of Industrial Organization, Cambridge, MA, MIT Press. 


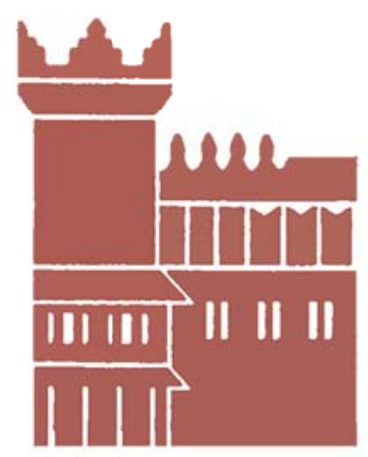

Alma Mater Studiorum - Università di Bologna DEPARTMENT OF ECONOMICS

Strada Maggiore 45

40125 Bologna - Italy

Tel. +39051 2092604

Fax +390512092664

http://www.dse.unibo.it 